\title{
Brain Diseases and Fall-Related Surgery in Older Persons
}

\author{
Ryuji Sakakibara ${ }^{a}$ Ayako limura ${ }^{a}$ Tsuyoshi Ogata ${ }^{a}$ Keiichiro Terayama ${ }^{a}$ \\ Shuichi Katsuragawa $^{a}$ Takaaki Nagao $^{a}$ Keiko Suzuki ${ }^{a}$ Kaori Izawa ${ }^{a}$ \\ Kiwa Nakajima $^{a}$ Shin-ichi Haruki ${ }^{a}$ Fuyuki Tateno $^{a}$ Yosuke Aiba ${ }^{a}$ \\ Masaaki Nemoto ${ }^{a}$ Koichi Nakagawa ${ }^{b}$
}

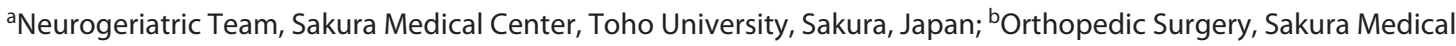

Center, Toho University, Sakura, Japan

\section{Keywords}

Subdural hematoma $\cdot$ Hip fracture $\cdot$ White matter disease $\cdot$

Alzheimer's disease · Dementia with Lewy bodies

\begin{abstract}
Background: It is known that age-related brain symptoms (gait difficulty and dementia) increase the likelihood of fallrelated surgery. In contrast, it is not known which types of brain disease underlie such symptoms most. Objective: The aim of this study was to correlate brain diseases with the types of surgeries performed at our hospital for patients who had fallen. Methods: This was a retrospective study at a multifaculty university hospital in Japan, with a 12-month recruiting period, a follow-up period of $3.0 \pm 2.5$ weeks, and $\geq 1 \times /$ week visits. We assembled a neurogeriatric team to diagnose brain diseases with the use of brain imaging to the extent possible and correlated the diagnoses with types of fall-related surgery. Results: Fall-related surgery was conducted by the orthopedics (OP) and neurosurgery (NS) faculties (total $n=124$ ) at a ratio of about 2 to 1 . The underlying brain diseases differed by faculty; for OP, surgery was most commonly performed in patients with a combination of white matter disease (WMD) and Alzheimer's disease (AD) (79\%) followed by dementia with Lewy bodies. In contrast,
\end{abstract}

for NS, the most common surgery was for patients with alcoholism (50\%) followed by a combination of WMD and AD. Conclusion: Fall-related surgery was performed by the OP and NS faculties at a 2 to 1 ratio. The major underlying brain diseases were a combination of WMD and AD (79\%) for OP and alcoholism (50\%) for NS.

(c) 2022 The Author(s)

Published by S. Karger AG, Basel

\section{Introduction}

It is known that age-related brain symptoms (encephalopathy, gait difficulty [Parkinsonism with/without sarcopenia] [1], and dementia/delirium [2]) increase the likelihood of fall-related surgery. In contrast, it is not known which types of brain disease underlie such symptoms most, although fall-related surgery is conducted in up to $51 \%$ of patients white matter disease (WMD, also called small-vessel disease, accounting for gait difficulty) [3-5], up to $55 \%$ of patients with Alzheimer's disease (AD, for dementia) $[6,7]$, up to $60 \%$ of patients with dementia with Lewy bodies (DLB, for gait difficulty and dementia) $[8,9]$, and up to $25.7 \%$ of patients with alcoholism (for gait difficulty and dementia) $[10,11]$. The reasons for this ambiguity may include as follows: (1) few

Correspondence to:

Ryuji Sakakibara, sakakibara@ sakura.med.toho-u.ac.jp

Karger@karger.com www.karger.com/ene

Karger $\stackrel{\text { ' }}{=}$
2022 The Author(s).

Published by S. Karger AG, Basel

This is an Open Access article licensed under the Creative Common Attribution-NonCommercial-4.0 International License (CC BY-NC) (http://www.karger.com/Services/OpenAccessLicense), applicable to the online version of the article only. Usage and distribution for commercial purposes requires written permission. 
studies have been performed to compare brain diseases and (2) few studies have looked at brain comorbidity (WMD and $\mathrm{AD}$, for example). In order to answer the above question, we developed a neurogeriatric team (neurologists, psychiatrists, neurosurgeons, nurses, neuropsychologists, pharmacologists, rehabilitation staff, and social workers) to diagnose brain diseases in order to provide advice and care for older patients [12] and correlated brain diseases with fall-related surgery.

\section{Patients and Methods}

This was a retrospective study with a 12 -month recruiting period, a follow-up period of $3.0 \pm 2.5$ (mean \pm standard deviation) weeks, and more than once-a-week visit. The inclusion criteria were (1) inpatient at a multifaculty university hospital in Japan, (2) a diagnosis of encephalopathy resulting in a referral to the neurogeriatric team [12], and (3) fall-related surgery or a plan to undergo such surgery. The referral was made because of the following points: (a) gait difficulty severe enough to require manual assistance or a wheelchair or (b) dementia/delirium severe enough for a positive score on the Clinical Dementia Rating scale [10] and/or the Confusion Assessment Method, either of which we consider "elderly encephalopathy." We added the Mini-Mental State Examination and the Frontal Assessment Battery to the extent possible. The criteria for preexisting elderly brain diseases were (a) WMD by the NINDS-AIREN criteria [13] (we added $>$ grade 2/4 WMD on Brant Zawadzki's magnetic resonance imaging (MRI)/ computed tomography (CT) grading scale to the extent possible in order to increase diagnostic accuracy and avoid obscurity) [14]; (b) probable AD by the NINCDS-ADRDA criteria [15] (we added $>$ grade $2 / 4$ para-hippocampus atrophy on the MRI/CT grading scale developed by Urs et al. [16] for the same reason); (c) probable DLB by the DLB Consortium criteria [17] (we added positivity on metaiodobenzylguanidine myocardial scintigraphy and/or dopamine transporter scan to the extent possible for the same reason); or (d) others (conforming to the published criteria) including acute/chronic subdural hematoma, alcoholism, normal-pressure hydrocephalus, prion disease, brain tumor/metastasis, subarachnoid hemorrhage, stroke, elderly-onset epilepsy, or any combination thereof. Exclusion criteria were (1) preexisting peripheral causes of vertigo (Ménière's disease and paroxysmal positional vertigo) or central causes of vertigo and (2) coma or terminally ill state. We did not have autopsy results. Statistical analyses were conducted with Student's $t$ test and the $\chi^{2}$ test. Each patient or a family member gave informed consent before the patient was accepted for care by the neurogeriatric team.

\section{Results}

We admitted a total of 11,134 patients to our hospital during the 12-month study period (15 faculty wards), of whom 5,762 (51.8\%) were elderly (age $\geq 65$ years). Fallrelated surgery was performed by the orthopedics (OP) and neurosurgery (NS) faculties (total $n=124,40$ men, 84 women, mean age 83.3 years), with the ratio of OP to NS being around 2 to 1 (84 [0.8\%] vs. 40 [0.4\% of hospital admissions, respectively]) (Fig. 1a). Most subjects had fallen from ground level, mostly at home. Only NS patients had fall-related surgery twice in the year (4 [10\% of NS], all men, all alcoholism/dementia as described below).

For OP ( $n=84$ [12\% of 699 faculty admissions], 6 men, 78 women, mean age 84.3 years), all fall-related surgeries were for the hip (femoral trochanteric or neck fracture). Among them, 8 (10\%) were aged $<65(40-64)$ years, while 76 (90\%) were aged $\geq 65$ (65-94) years. Preexisting factors other than age-related encephalopathy were largely unknown. The 8 patients aged $<65$ years old included one who had a traffic-related bike accident, one who had a job-related auto accident, and one who had a skateboard accident. Regarding the relationship between encephalopathy and age, one of 8 (12.5\%, man, alcohol cirrhosis and dementia) aged $<65$ years had encephalopathy, while 70 of 76 (92\%) aged $\geq 65$ years had age-related encephalopathy. The most common underlying brain diseases for the 70 encephalopathy cases in OP were a combination of WMD and AD in 56 (79\%); DLB in 10; WMD, $\mathrm{AD}$, and DLB in 2; WMD and idiopathic normal-pressure hydrocephalus in 1; WMD and DLB in 1; and alcohol cirrhosis and dementia in 1 (Fig. 1b). Parkinson's disease without dementia or other movement disorders were not found. Seven of them had treated diabetes, and none had marked polyneuropathy.

For NS ( $n=40$ [12\% of 367 faculty admissions], 34 men, 6 women, mean age 78.8 years), 40 fall-related surgeries were performed (acute, 7 , chronic, 33, of subdural hematoma). Among them, 5 (11.4\%) were aged <65 (51-64) years, while $35(88.6 \%)$ were aged $\geq 65(65-94)$ years. Regarding the relationship between encephalopathy and age, one of $5(20 \%)$ with age $<65$ years had encephalopathy, while 33 of 35 (94\%) with age $\geq 65$ years had encephalopathy. Preexisting factors other than age-related encephalopathy were largely unknown, while in 5 aged $<65$ years, all had alcoholism/dementia. The most common underlying brain disease for 34 encephalopathy cases in NS were alcoholism/dementia in 17 (50\%) (alcoholism in 9; alcoholism, WMD, and AD in 4; alcoholism and WMD in 3; and alcoholism and DLB in one), followed by a combination of WMD and AD in 15 (44\%) and DLB in 2 (Fig. 1b). There were no cases of co-necessity of OP and NS surgeries in our cohort. Parkinson's disease without dementia or other movement disorders were not found. Three of them had treated diabetes, and none had marked polyneuropathy.

In summary, 115 (93\%) of 124 fall-related surgery patients were elderly (age $\geq 65$ years), of whom 103 (90\%) 
Fig. 1. Causes of fall-related surgery and contributions of brain diseases at a multifaculty university hospital. a Incidences of fall-related surgery types among annual total hospital admissions. surgery cases (femoral neck and trochanteric fracture) were more common than NS (subdural hematoma) cases. b Ratios of brain diseases underlying fall-related surgery. Major brain diseases in surgeries performed by OP faculty were a combination of WMD and $\mathrm{AD}$; those performed by NS faculty were alcoholism in combination with dementia. iNPH, idiopathic normal-pressure hydrocephalus.
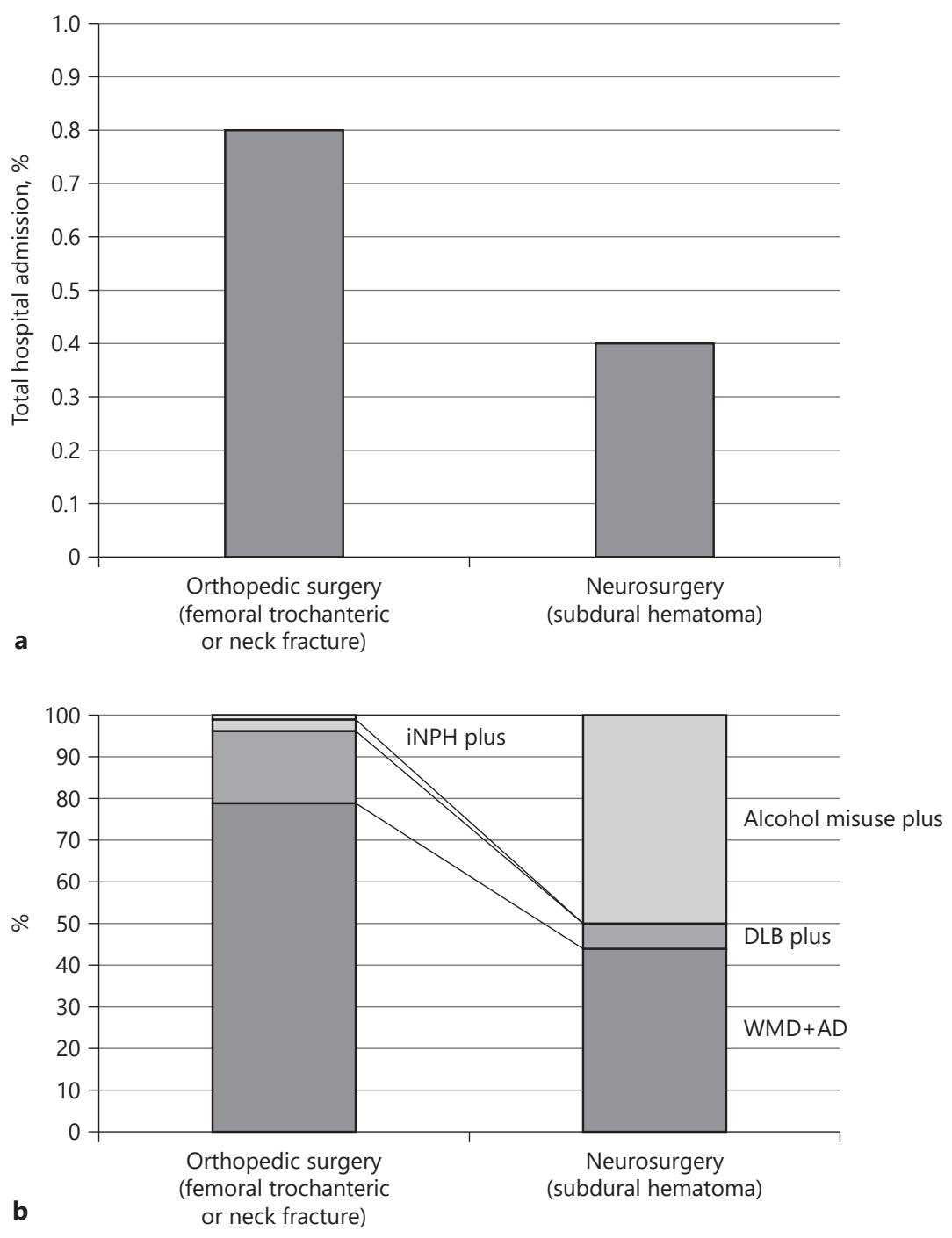

had age-related brain symptoms (encephalopathy). Further, the most common underlying brain disease was a combination of WMD and AD in 79 (75\%), followed by alcoholism/dementia in 18 (17\%) and DLB in 16 (15\%) (overlap counted). None of the differences in these values reached statistical significance.

\section{Discussion}

We assembled a neurogeriatric team to review fall-related surgeries performed at our multifaculty university hospital. Such surgeries were performed by the OP and
NS faculties at a ratio of around 2 to 1 . The ratio differs slightly from that reported in the US National Trauma Data Bank study by Ahmed et al. [18], which showed a ratio of around 1 to 1 (i.e., $16.7 \%$ vs. $19.9 \%$ ). We do not know the exact reason for this discrepancy. However, the potential bias of our study might be that our cohort included fewer cases of alcoholism (closely associated with subdural hematoma as described below) than the cohort in the US study.

Our study is unique in that we explored age-related brain diseases that lead to fall-related surgery. As a result, the most common brain disease was a combination of $\mathrm{WMD}$ and $\mathrm{AD}(75 \%)$, followed by alcoholism/dementia 
(17\%) and DLB (15\%) (overlap counted). These figures might reflect the frequency of elderly brain diseases in octogenarians, e.g., the ratio of WMD versus $A D$ versus $\mathrm{DLB} / \mathrm{PD}$ is approximately $80-33 \%$ to $8 \%$ [19]. Recently, it was also recognized that the prevalence of triple/dual disease (any combination of WMD, $\mathrm{AD}$, or $\mathrm{DLB}$ ) is up to $60 \%$ in octogenarians [19]. It is worth mentioning that the underlying brain diseases differ between OP surgeries and NS surgeries. For example, in OP, the most common diseases were a combination of WMD and AD (79\%) followed by DLB and others; in NS, on the other hand, the most common diseases were alcoholism/dementia (50\%) followed by a combination of WMD and AD and others. Alcoholism/dementia is a strong contributor to subdural hematoma $[11,12]$. This is probably because long-lasting alcoholism gives rise to gait difficulty (by cerebellar ataxia and peripheral neuropathy) $[11,12]$ together with dementia (including Wernicke-Korsakoff syndrome, postconcussion syndrome [those who did not realize they had fallen] [20], etc.). Similarly, DLB gives rise to gait difficulty (by Parkinsonian disorder) and dementia (commonly associated with delirium [21]) together with postural syncope [8-10]. All these factors are known to produce hip fracture in DLB $[11,12,22]$. In contrast, as described above, many older subjects have a combination of WMD and AD (dual disease) [19, 23, 24]. This combination of WMD and $\mathrm{AD}$ also produces gait disorder (by WMD) [3-5] together with dementia (by AD) [6, 7]. It is reported that dual diseases may worsen gait and cognitive performance to a greater extent than single disease [23, 24].

The present study had the following limitations. We did not include nonsurgical trauma such as hand fractures; therefore, the incidence of total falls seems likely to be higher than that reported in the present study. We diagnosed $\mathrm{AD}$ and WMD by neurogeriatric examination and brain MRI/CT. In contrast, we diagnosed DLB mainly by a neurogeriatric examination and could not perform metaiodobenzylguanidine myocardial scintigraphy or dopamine transporter scans for all patients because of their behavioral and psychological symptoms of dementia and acute confusion; the possibility of false-negative diagnoses of DLB therefore cannot be excluded. We did not evaluate cerebrospinal fluid biomarkers, and we did not have pathology results; thus, the possibility of falsepositive results cannot be excluded. Finally, we could not analyze factors of acute encephalopathy in this study. This includes the following factors: those for OP and NS, medications (anesthetics, analgesics, and sedatives); for $\mathrm{OP}$, systemic encephalopathy where tumor necrosis fac- tor-alpha is thought to penetrate the blood-brain barrier [25]; and for NS, acute brain insult itself [26].

In conclusion, fall-related surgeries were performed by the OP and NS faculties at a 2 to 1 ratio. The major underlying brain diseases in OP surgeries were a combination of WMD and AD (79\%) and that in NS surgeries was alcoholism (50\%).

\section{Statement of Ethics}

Each patient or a family member gave written informed consent before the patient was accepted for care by the neurogeriatric team in the present study. The study was approved by the Ethics Committee in Sakura Medical Center, Toho University (No. 2011059).

\section{Conflict of Interest Statement}

The authors have no conflict of interest to declare.

\section{Funding Sources}

We have no funds. None of the authors received financial support relevant to this study. No author has received any funding from any institution, including personal relationships, interests, grants, employment, affiliations, patents, inventions, honoraria, consultancies, royalties, stock options/ownership, or expert testimony in the last 12 months. No sponsors were involved in this study. The authors state that they have no conflicts of interest to declare.

\section{Author Contributions}

R. Sakakibara participated in the study concept and design; acquisition of patients; and/or data, analysis and interpretation of data, and preparation of the manuscript. A. Iimura, K. Terayama, S. Katsuragawa, T. Nagao, K. Suzuki, K. Izawa, K. Nakajima, Y. Aiba, M. Nemoto, K. Nakagawa, and T. Ogata participated in the acquisition of patients and/or data. S Haruki has nothing to declare and no conflict of interest. F. Tateno participated in the acquisition, analysis, and interpretation of data.

\section{Data Availability Statement}

All data generated or analyzed during this study are included in this article. Further inquiries can be directed to the corresponding author. 


\section{References}

1 Kim J, Jang SN, Lim JY. Pre-existing disability and its risk of fragility hip fracture in older adults. Int J Environ Res Public Health. 2019 Apr 7;16(7):1237.

2 Kennedy GEM, Mohandas P, Anderson LA, Kennedy M, Shirley DSL. Improving identification of cognitive impairment in fragility fracture patients: impact of educational guidelines on current practice. Geriatr $\mathrm{Or}$ thop Surg Rehabil. 2020 Jul 27;11: 2151459320935095

3 Sibolt G, Curtze S, Melkas S, Pohjasvaara T, Kaste M, Karhunen PJ, et al. White matter lesions are associated with hospital admissions because of hip-fractures and trauma after ischemic stroke. Stroke. 2014;45:2948-51.

4 Srikanth V, Beare R, Blizzard L, Phan T, Stapleton J, Chen J, et al. Cerebral white matter lesions, gait, and the risk of incident falls: a prospective population-based study. Stroke. 2009;40:175-80.

5 Koo BB, Bergethon P, Qiu WQ, Scott T, Hussain M, Rosenberg I, et al. Clinical prediction of fall risk and white matter abnormalities: a diffusion tensor imaging study. Arch Neurol. 2012;69:733-8

6 Roh HW, Hong CH, Lee SJ, Lee Y, Lee KS, Chang KJ, et al. Frontal lobe function and risk of hip fracture in patients with Alzheimer disease: an analysis of linked data. Medicine. 2015 Nov;94(45):e1918.

7 Oh ES, Blennow K, Bigelow GE, Inouye SK, Marcantonio ER, Neufeld KJ, et al. Abnormal CSF amyloid- $\beta 42$ and tau levels in hip fracture patients without dementia. PLoS One. 2018 Sep 25;13(9):e0204695.

8 Hanyu H, Sato T, Hirao K, Kanetaka H, Sakurai $\mathrm{H}$, Iwamoto $\mathrm{T}$. Differences in clinical course between dementia with Lewy bodies and Alzheimer's disease. Eur J Neurol. 2009; 16:212-7.

9 Enemark M, Midttun M, Winge K. Evaluating outcomes for older patients with Parkinson's disease or dementia with lewy bodies who have been hospitalised for hip fracture surgery: potential impact of drug administration. Drugs Aging. 2017;34:387-92.
10 Sousa EB, Brandão LF, Tavares CB, Borges IB, Neto NG, Kessler IM. Epidemiological characteristics of 778 patients who underwent surgical drainage of chronic subdural hematomas in Brasília, Brazil. BMC Surg. 2013 Mar 1;13:5.

11 Marley WD, Kelly G, Thompson NW. Alcohol-related fracture admissions: a retrospective observational study. Ulster Med J. 2015; 84:94-7.

12 Sakakibara R, Katsuragawa S, Iimura A, Ogata T, Terayama K, Doi H, et al. Neurogeriatric approach to delirium/dementia in a multifaculty university hospital. Neurol Clin Neurosci. 2020;9:68-72.

13 Román GC, Tatemichi TK, Erkinjuntti T, Cummings JL, Masdeu JC, Garcia JH, et al. Vascular dementia: diagnostic criteria for research studies. Report of the NINDS-AIREN International Workshop. Neurology. 1993; 43:250-60.

14 Brant-Zawadzki M, Fein G, Van Dyke C, Kiernan R, Davenport L, de Groot J. MR imaging of the aging brain: patchy white-matter lesions and dementia. AJNR Am J Neuroradiol. 1985;6:675-82.

15 McKhann G, Drachman D, Folstein M, Katzman R, Price D, Stadlan EM. Clinical diagnosis of Alzheimer's disease: report of the NINCDS-ADRDA Work Group under the auspices of department of health and human services task force on Alzheimer's disease. Neurology. 1984;34:939-44.

16 Urs R, Potter E, Barker W, Appel J, Loewenstein DA, Zhao W, et al. Visual rating system for assessing magnetic resonance images: a tool in the diagnosis of mild cognitive impairment and Alzheimer disease. J Comput Assist Tomogr. 2009;33:73-8

17 McKeith IG, Boeve BF, Dickson DW, Halliday G, Taylor JP, Weintraub D, et al. Diagnosis and management of dementia with Lewy bodies: fourth consensus report of the DLB consortium. Neurology. 2017;89:88-100.
18 Ahmed N, Greenberg P. Early risk stratification of in hospital mortality following a ground level fall in geriatric patients with normal physiological parameters. Am J Emerg Med. 2020;38(12):2531-5.

19 Sakakibara R, Tateno F, Aiba Y, Ogata T, Terada H, Inaoka T, et al. Prevalence of triple/ dual disease (Alzheimer's disease, Lewy body disease, and white matter disease). Neurol Clin Neurosci. 2020;8:171-6.

20 Ganti L, Khalid H, Patel PS, Daneshvar Y, Bodhit AN, Peters KR. Who gets post-concussion syndrome? An emergency department-based prospective analysis. Int J Emerg Med. 2014 Aug 20;7:31.

21 Vardy E, Holt R, Gerhard A, Richardson A, Snowden J, Neary D. History of a suspected delirium is more common in dementia with Lewy bodies than Alzheimer's disease: a retrospective study. Int J Geriatr Psychiatry. 2014;29:178-81.

22 Kudo Y, Imamura T, Sato A, Endo N. Risk factors for falls in community-dwelling patients with Alzheimer's disease and dementia with Lewy bodies: walking with visuocognitive impairment may cause a fall. Dement Geriatr Cogn Disord. 2009;27:139-46.

23 Ogama N, Sakurai T, Shimizu A, Toba K. Regional white matter lesions predict falls in patients with amnestic mild cognitive impairment and Alzheimer's disease. J Am Med Dir Assoc. 2014;15:36-41.

24 Sakakibara R, Tateno F, Aiba Y, Ogata T, Kishi $\mathrm{M}$, Terada $\mathrm{H}$, et al. Prevalence and clinical features of dual diseases of Alzheimer's disease and dementia with Lewy bodies: a neuroimaging-based study. Neurol Clin Neurosci. 2020;8:171-6.

25 Manian FA, Hsu F, Huang D, Blair A, Mosarla R, Mulugeta W, et al. Coexisting systemic infections in patients hospitalized because of a fall: prevalence and risk factors. J Emerg Med. 2020;58:733-40.

26 Black DW. Mental changes resulting from subdural haematoma. Br J Psychiatry. 1984; 145:200-3. 